\title{
Epileptic Encephalopathies in Infants and Children: Study of Clinico-Electroencephalographic Spectrum in a Tertiary Hospital in Bangladesh
}

\author{
Bithi Debnath ${ }^{*}$, Rajib Nayan Chowdhury ${ }^{2}$, Narayan Chandra Shaha ${ }^{1}$, \\ Mohammad Enayet Hussain ${ }^{2}$
}

${ }^{1}$ Department of Paediatric Neurology, National Institute of Neurosciences and Hospital, Sher-E-Bangla Nagar, Dhaka, Bangladesh ${ }^{2}$ Department of Neurophysiology, National Institute of Neurosciences and Hospital, Sher-E-Bangla Nagar, Dhaka, Bangladesh Email: *bithidebnath@gmail.com, rajibnayandr@gmail.com,91janm@gmail.com, enayetdmc@yahoo.com

How to cite this paper: Debnath, B., Chowdhury, R.N., Shaha, N.C. and Hussain, M.E. (2021) Epileptic Encephalopathies in Infants and Children: Study of Clinico-Electroencephalographic Spectrum in a Tertiary Hospital in Bangladesh. Open Journal of Pediatrics, 11, 339-350. https://doi.org/10.4236/ojped.2021.113031

Received: June 1, 2021

Accepted: July 12, 2021

Published: July 15, 2021

Copyright $\odot 2021$ by author(s) and Scientific Research Publishing Inc. This work is licensed under the Creative Commons Attribution International License (CC BY 4.0).

http://creativecommons.org/licenses/by/4.0/

\section{Abstract}

Background: The epileptic encephalopathies collectively exact an immense personal, medical, and financial toll on the affected children, their families, and the healthcare system. Objective: This study was aimed to delineate the clinical spectrum of patients with Epileptic encephalopathies (EEs) and classify them under various epileptic syndromes. Methods: This was a cross-sectional study that was carried out in the department of Neurophysiology of the National Institute of Neurosciences and Hospital, Bangladesh from July 2016 to June 2019 . Children with recurrent seizures which were difficult to control and associated with developmental arrest or regression in absence of a progressive brain pathology were considered to be suffering from EE. Children under 12 years of age fulfilling the inclusion criteria were enrolled in the study. These patients were evaluated clinically and Electroencephalography (EEG) was done in all children at presentation. Based on the clinical profile and EEG findings the patients were categorized under various epileptic syndromes according to International League Against Epilepsy (ILAE) classification 2010. Results: A total of 1256 children under 12 years of age were referred to the Neurophysiology Department. Among them, 162 $(12.90 \%)$ fulfilled the inclusion criteria. Most of the patients were male (64.2\%) and below 1 year (37.7\%) of age. The majority (56.8\%) were delivered at the hospital and $40.1 \%$ had a history of perinatal asphyxia. Development was age-appropriate before the onset of a seizure in $38.9 \%$ of cases. Most (53.7\%) of the patients had seizure onset within 3 months of age. Categorization of Epileptic syndromes found that majority had West Syndrome (WS) 
(37.65\%) followed by Lennox-Gastaut syndrome (LGS) (22.22\%), Otahara syndrome (11.73\%), Continuous spike-and-wave during sleep (CSWS) (5.66\%), Myoclonic astatic epilepsy (MAE) (4.94\%), Early myoclonic encephalopathy (EME) (3.7\%), Dravet syndrome (3.7\%) and Landau-Kleffner syndrome (LKS) (1.23\%). 9.26\% of syndromes were unclassified. Conclusion: EEG was found to be a useful tool in the evaluation of Epileptic encephalopathies. The clinico-electroencephalographic features are age-related. Their recognition and appropriate management are critical.

\section{Keywords}

Epileptic Encephalopathy (EE), EEG, Infantile Epileptic Encephalopathy (IEE), Clinico-Electroencephalographic Spectrum, West Syndrome

\section{Introduction}

Epileptic encephalopathy (EE) refers to a category of conditions in which epileptic activity itself can lead, and can intensify over time, to serious cognitive and behavioral impairments beyond what can be expected from the underlying pathology alone (e.g., cortical malformation). Such impairments can be more selective or global, and they can occur along a severity continuum. Some syndromes are sometimes referred to as epileptic encephalopathies (EEs), with the potential for encephalopathic effects of seizures and epilepsy associated with any type of epilepsy [1]. It may be progressive or it may have a direction that fluctuates. Some syndromes are recognized [Early myoclonic encephalopathy (EME), Ohtahara syndrome or Early Infantile Epileptic Encephalopathy (EIEE), West syndrome (WS), Dravet syndrome, Lennox-Gastaut syndrome (LGS), Landau-Kleffner syndrome (LKS), Epileptic encephalopathy with continuous spike-and-wave during sleep (CSWS)] while some (Epilepsy of infancy with migrating focal seizures, Atypical benign partial epilepsy of childhood, Hypothalamic epilepsy, Late infantile epileptic encephalopathy, Myoclonic encephalopathy in nonprogressive disorders) have been proposed for inclusion under EEs [2].

The clinical spectrum of EEs is complex and depends on the age of onset, epileptic behavior, environmental and genetic variables [3]. The clinical spectrum varies from severe forms with cognitive and motor deterioration to mild forms with a better course [4]. Clinical and electroencephalographic (EEG) characteristics represent the immature brain's particular age-related epileptogenic reaction. Several studies have shown that better control of seizures leads to an improvement of the developmental outcome in certain children [5] [6]. However, despite aggressive and sometimes off-label use of several medications, the response to anticonvulsants is often poor [7].

In every paediatric neurology clinic, they are major contributors to neuro morbidity-physical, social, intellectual disability, and intractable seizures. Be- 
cause of their refractoriness and poor cognitive outcome, even though seizures are controlled, there has been a recent emphasis on their further classification and management [8]. More vigorous use of antiepileptic drugs (AEDs) in the suppression of interictal epileptiform discharges is considered successful. Besides AEDs, immunomodulatory therapies (e.g., corticosteroids, IVIG, plasmapheresis) and the Ketogenic or Adkin's diet are often considered.

In this study, we analyzed and categorized the electro-clinical spectrum of children with EEs into different epileptic syndromes. Their clinical and seizure spectrum, perinatal causes, and EEG results were addressed. However, overlapping clinico-electrical characteristics often make it difficult to classify patients into a particular syndrome.

\section{Material and Methods}

This was a hospital-based cross-sectional observational study. This study was conducted in the department of Neurophysiology of the National Institute of Neurosciences and Hospital, Dhaka, Bangladesh during the period from July 2016 to June 2019. Children below 12 years of age referred from the epilepsy clinic of the pediatric neurology department were screened.

Children who had a recurrent seizure, not controlled by two or more antiepileptic drugs with adequate dose and duration having severe cognitive and behavioral impairments not explained by the underlying pathology alone were considered to have EEs and enrolled. These children usually had multiple seizure types and seizures could directly worsen their cognition and behavior. Patients with subacute sclerosing panencephalitis were excluded. A convenient consecutive sampling method was applied and data were collected in a pre-designed questionnaire. These children were evaluated by history and physical findings. The neurodevelopmental assessment was done by a Developmental therapist using Rapid neurodevelopmental assessment [9] [10] tools. EEG was done in all children at the presentation where surface electrodes were distributed according to the 10 - 20 International System where impedances were kept at or below 5000 ohms. EEG and EKG were recorded simultaneously. EEG recordings were taken during spontaneous sleep and awake state for about 60 mins.

Age of presentation and seizure onset, seizure spectrum, developmental profile, perinatal events including mode of delivery, perinatal asphyxia, Central nervous system infection, neonatal jaundice, family history of seizure, microcephaly, EEG abnormalities, brain imaging (CT, MRI) findings were evaluated. Name of the antiepileptic drugs (AEDs), response to ACTH or other drugs were also noted.

Considering the age of onset of the seizure, its semiology and evolution, interictal condition, and EEG findings, the patients were categorized under various epileptic syndromes according to International League Against Epilepsy (ILAE) classification 2010. Despite the evolution from one form of EE to another, the categorization which was done on enrolment was retained for the study. Child- 
ren with EEs were classified into WS, LGS, EME, EIEE, Dravet syndrome, LKS, MAE, and CSWS. When electro-clinical classification could not possible, it remained unclassified.

The criteria for various EEs in this study were [4] [11]:

Ohtahara syndrome (EIEE): Age of onset ranging from birth to 3 months of age, high-frequency tonic spasms are the defining seizure type, other seizure types also occur; burst suppression pattern without sleep-wake differentiation on interictal EEG.

Early myoclonic encephalopathy (EME): Seizure onset in neonatal period, erratic, fragmentary, or massive myoclonus, focal seizures, and late tonic spasms; EEG pattern is bursts-suppression with long suppression periods; more distinct during sleep.

West syndrome (WS): Cluster of spasms started within 3 to 12 months of age, psychomotor deterioration, and hypsarrhythmia on EEG.

Lennox-Gastaut syndrome (LGS): Age of onset ranging from 1 to 8 years of age, refractory polymorphic seizures ( $\geq 2$ seizure types, the tonic seizure must be one seizure type), on EEG-bilateral synchronous slow spike-and-wave discharges $(1.5-2.5 \mathrm{~Hz}$ ) with abnormal slow background and/or paroxysmal fact activity.

Dravet syndrome: Age of onset between 5 and 8 months, history of febrile seizure followed by afebrile multiple seizure types in a previously normal child, psychomotor regression; rhythmic theta $(4-5 \mathrm{~Hz})$ activity and generalized photo paroxysmal responses, slow background, multifocal epileptiform discharges or asymmetric paroxysms of generalized poly-spike/spike-slow waves on EEG.

Landau-Kleffner syndrome (LKS): Age of onset ranging from 5 to 8 years, developmentally age-appropriate children presented with verbal auditory agnosia, language regression, behavioral abnormalities; tonic-clonic, focal motor, atypical absences, head drop, and subtle seizures; posterior temporal (vertical dipole) epileptiform discharges markedly activated by NREM sleep on EEG.

Myoclonic astatic epilepsy (MAE): Seizure onset between 2 and 5 years of age, multiple seizure types (myoclonic-astatic, tonic-clonic, tonic, and atypical absences; apraxia, dysarthria, and cognitive deterioration; poly-spike waves or spike-wave complexes at a frequency of $2-4 \mathrm{~Hz}$ on ictal EEG, spike-waves of 3 $\mathrm{Hz}$ may appear later and non-convulsive status may be seen on EEG.

Continuous spike-and-wave during sleep (CSWS): Age of ranging from 4 to 7 years; multiple seizure types; psychomotor and speech regression, behavioral abnormality and motor involvement (ataxia, dystonia, dyspraxia) are often present; continuous spike-wave complexes exclusively during non-REM sleep, with a spike-wave index for at least $80-85 \%$ of slow sleep on EEG.

All data were entered in SPSS 16 for Windows statistical software. Continuous data were presented as means and standard deviations whereas categorical data were presented as proportions. Written informed consent for enrollment was taken from every parent. Ethical clearance from the ethical review committee (ERC) of the hospital was taken before starting the study. 


\section{Results}

A total of 1256 patients under 12 years were referred to Neurophysiology Department for EEG from July 2016 to June 2019. Among them, 162 (12.90\%) fulfilled the inclusion criteria. Their mean age was $32.43(1-120)$ days. Most of the patients were male $(64.2 \%)$ and below 1 year $(37.7 \%)$ of age (Table 1$)$.

Most (56.8\%) were delivered at term (82.7\%) at the hospital (56.8\%) and cesarean section was done only in $39.51 \%$ of cases. $40.1 \%$ had a history of perinatal asphyxia. Development was age-appropriate before the onset of a seizure in $38.9 \%$ of cases. In $13.3 \%$ of cases, there was a family history of seizures (Table 2).

Most (53.7\%) of patients had seizure onset within 3 months of age (Figure 1) and on neurodevelopmental assessment, the majority (96.9\%) had a developmental

Table 1. Age and sex distribution of patients $(n=162)$.

\begin{tabular}{ll}
\hline Sex & Male: $104(64.2 \%)$ \\
\hline Mean age (month) & Female: $58(35.8 \%)$ \\
\hline & $32.43(1-120)$ \\
\hline Age at presentation (month) & $1-3: 11(6.8 \%)$ \\
& $4-12: 50(30.9 \%)$ \\
& $13-36: 48(29.6 \%)$ \\
& $37-60: 24(14.8 \%)$ \\
& $>60: 29(17.9 \%)$ \\
\hline
\end{tabular}

Table 2. Perinatal and developmental profile of the studied population $(n=162)$.

\begin{tabular}{lcc}
\hline \multicolumn{1}{c}{ Variables } & Number & Percentage (\%) \\
\hline Gestational age & 135 & 82.7 \\
Term & 19 & 11.7 \\
Preterm & & \\
Place of delivery & 92 & 56.8 \\
Hospital & 70 & 43.2 \\
Home & & \\
Mode of delivery & 98 & 60.49 \\
Normal vaginal delivery & 64 & 39.51 \\
Cesarean section & & \\
Etiology & 65 & 40.1 \\
History of perinatal asphyxia & 36 & 22.22 \\
CNS infection & 27 & 16.67 \\
Septicemia & 10 & 6.17 \\
Bilirubin encephalopathy & 63 & 38.9 \\
Development age-appropriate before the onset of seizure & 22 & 13.3 \\
Family history of seizure & & \\
\hline
\end{tabular}


delay either global or at specific domains. $23.4 \%$ of patients had microcephaly (Table 3).

Infantile spasm (45.1\%) and myoclonic seizures (40.1\%) were the commonest types of seizures (Table 4). EEG was found to be abnormal in $93.2 \%$ of patients on enrollment. The most common EEG abnormality was multifocal epileptiform discharges (31.9\%) and generalized epileptiform discharges (27.5\%) (Table 5).

Electroclinical diagnosis established WS (37.65\%) to be the most common followed by LGS (22.22\%), EIEE (11.73\%), CSWS (5.56\%), MAE (4.94\%), EME (3.7\%), Dravet (3.7\%) and LKS (1.23\%). 9.26\% of children having EE remained unclassified (Figure 2). Sodium valproate was the most commonly prescribed drug and $18.02 \%$ of patients were on monotherapy.

Table 3. Physical findings of the studied population on enrollment $(n=162)$.

\begin{tabular}{lcc}
\hline \multicolumn{1}{c}{ Variables } & Number & Percent (\%) \\
\hline Developmental delay & 157 & 96.9 \\
Tone abnormalities & 66 & 40.7 \\
Quadriplegia & 42 & 25.9 \\
Microcephaly & 38 & 23.4 \\
Visual deficit & 22 & 13.5 \\
Hemiplegia & 9 & 5.5 \\
Hearing deficit & 7 & 4.3 \\
\hline
\end{tabular}

Table 4. Seizure spectrum of the studied population $(n=162)$.

\begin{tabular}{lccc}
\hline \multicolumn{1}{c}{ Seizure semiology } & Number & Percent (\%) & Percent (\%) of \\
cases
\end{tabular}

Table 5. EEG findings in the studied population $(n=162)$.

\begin{tabular}{lccc}
\hline \multicolumn{1}{c}{ EEG findings } & Number & Percent (\%) & Percent (\%) of cases \\
\hline Multifocal epileptiform discharge & 44 & 27.7 & 31.9 \\
Generalized epileptiform discharge & 38 & 23.9 & 27.5 \\
Hypsarrhythmia & 20 & 12.6 & 14.5 \\
Focal epileptiform discharge & 15 & 9.4 & 10.9 \\
Burst suppression in both awake \& sleep & 14 & 8.8 & 10.1 \\
Modified hypsarrhythmia & 13 & 8.2 & 9.4 \\
Generalized paroxysmal fast activity & 10 & 6.3 & 7.2 \\
Burst suppression predominantly & & 3.1 & 3.6 \\
in sleep & 5 & & \\
\hline
\end{tabular}




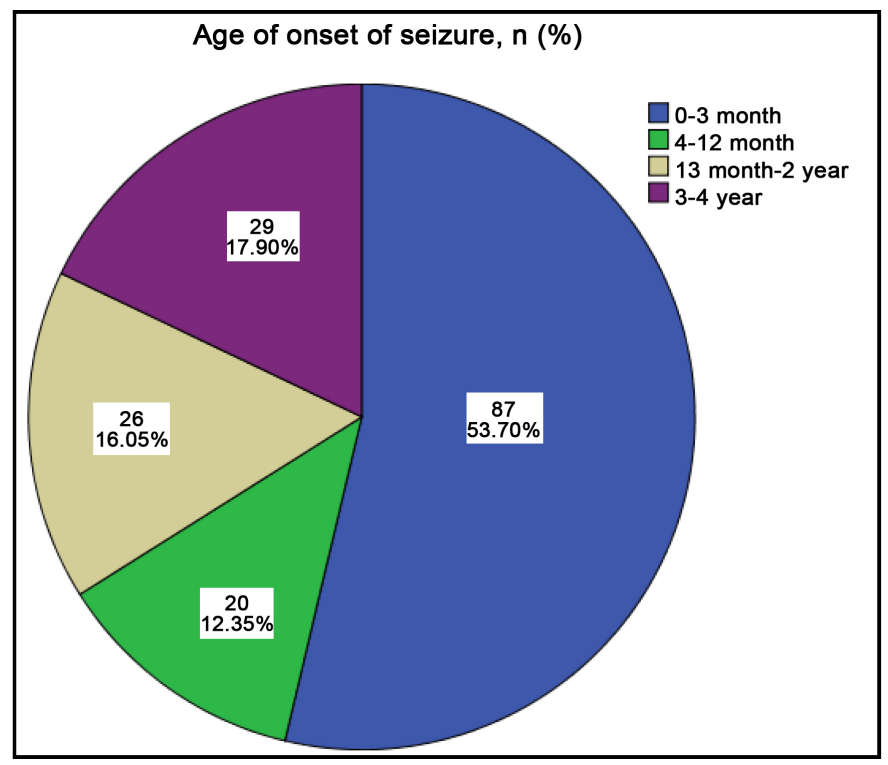

Figure 1. Age of onset of seizure $(\mathrm{n}=162)$.

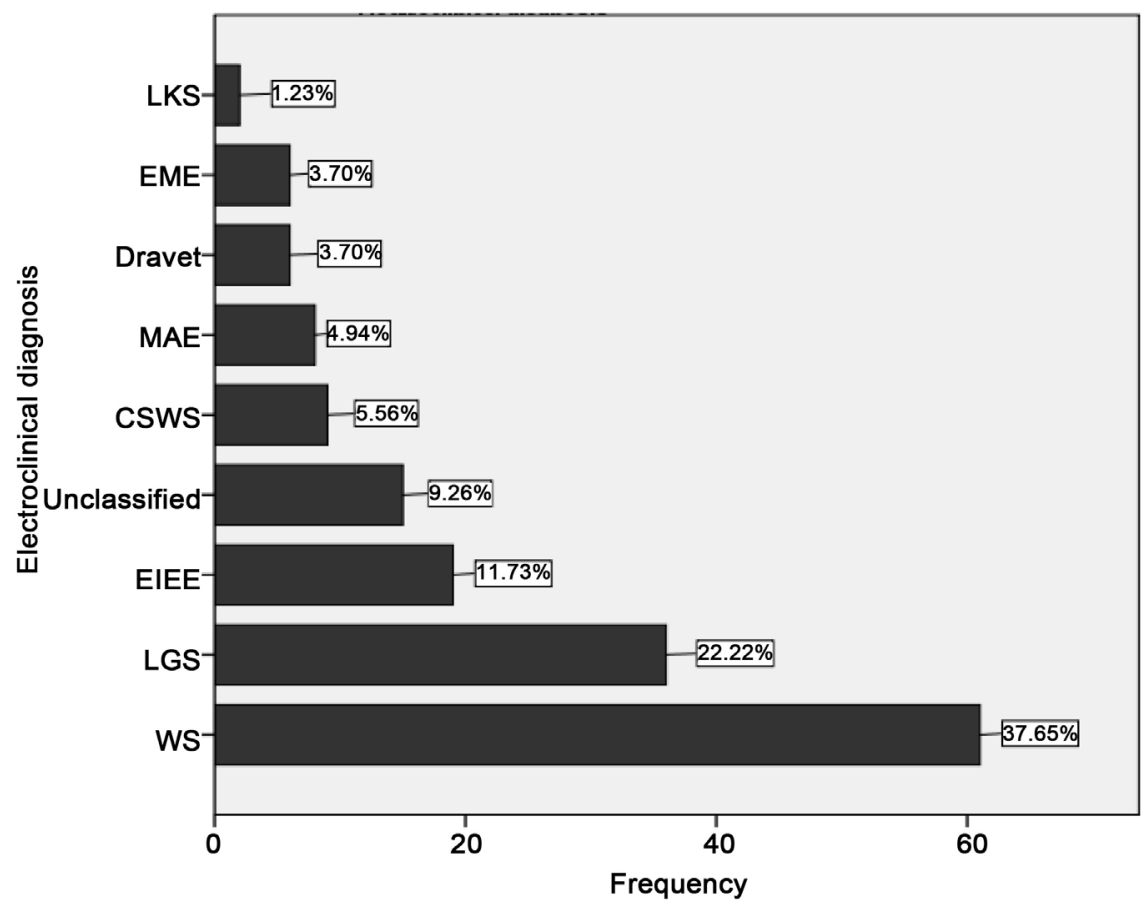

Figure 2. Electroclinical diagnosis among the studied population $(\mathrm{n}=162)$. WS-West syndrome, LGS-Lennox-Gastaut syndrome, EIEE-Early Infantile Epileptic Encephalopathy, CSWS-Continuous spike wave of slow sleep, MAE-Myoclonic-astatic epilepsy, Dravet-Dravet syndrome, EME_Early myoclonic encephalopathy, LKS_Landau-Kleffner syndrome.

\section{Discussion}

EEs are an age-related diverse group of disorders where seizure control becomes very difficult and there is either developmental arrest or regression. It has a distinctive seizure pattern and specific EEG abnormalities. It may be progressive or 
may have a waxing-waning course. However, syndromic classification is often difficult. These conditions can overlap and develop into other clinical and electroencephalographic characteristics. In the literature [12] [13] [14], this is well known. These kids also switch from one doctor to another without a correct diagnosis and receiving several medications. For better results, these patients need early diagnosis and intensive care. The task of electroclinical classification is therefore extremely important for the proper management of EEs in these patients.

This study included $162(12.90 \%)$ patients with suspected epileptic encephalopathy. Males were almost twice the number of female patients. This finding is similar to the findings in other studies [11] [15]. Although only $6.8 \%$ of patients presented at 1 to 3 months of age, half of the enrolled children had onset of seizure within 3 months of age. Therefore, there was a delay between the onset of symptoms and disease recognition. Earlier the onset of seizures more is the damage [14]. A history of perinatal asphyxia was present in around $40 \%$ of patients. Perinatal asphyxia emerged as an important etiological factor in many of the EEs in different studies [11] [16]-[23]. In this study, there was a history of central nervous system infection in early infancy in $22.22 \%$ of cases. This finding differs from the data of developed countries where most of the cases are due to prenatal causes [24] [25].

In all EEs, developmental delay or arrest is a prime concern. In our study, it was observed at initial presentation in $96.9 \%$ of cases which is comparable to literature (90\%) [13] and another study [11]. In this study, 38.9\% of children with EEs were developmentally age-appropriate before the onset of a seizure and many of them became retarded at the time of enrollment in the study. Microcephaly was found in $23.3 \%$ of patients which is less than other previous studies [11] [17] [26] and to a figure mentioned in literature [27]. An earlier cerebral insult like intrauterine infections, asphyxia, metabolic, genetic, or developmental malformation of the brain may cause microcephaly [11].

In a previous study, WS and EIEE were found to be the most common and constitute almost half of all EEs [11]. Most of the patients of EIEE and WS presents in the first three months of their life with seizures. In this case series, seizures started within the first 3 months of age among most patients and the WS was the most frequent EE. Infantile spasms and myoclonic seizures were the commonest type of seizures followed by tonic and atonic seizures. As WS and LGS constitute the majority of cases in this series, these seizures are expected to be the most frequent seizure types. Most of the EEGs were abnormal; multifocal and generalized epileptiform discharges were the commonest abnormality. Multiple independent epileptiform discharges have been considered important EEG findings in many of the EEs [28] [29]. EEG recordings allow early detection of EEs thereby facilitating immediate aggressive treatment.

In a national household survey in Bangladesh, it is shown that the prevalence of epilepsy among children aged $<18$ years is 8.2 per 1000 [30]. So it is a huge burden for a developing country like us. A study conducted in Dhaka Shishu 
(Children's) Hospital among children with epilepsy aged 2 months to 15 years found "Severe epilepsy syndrome" in 31\% of cases. Where WS (66\%) was the commonest followed by Myoclonic encephalopathy (24\%), LGS (9\%), and LKS (1\%) [16]. Kalra et al. [11] in a study in the All India Institute of Medical Sciences observed that Infantile epileptic encephalopathies (IEE) comprised $3.5 \%$ of Paediatric Neurology Clinic registrations. WS was the commonest IEE and comprised 55.3\%. EIEE, LGS, and EME were found in $26.6 \%, 16 \%$, and $2.1 \%$ cases respectively. In our study, we found $12.90 \%$ of children ( $>12$ years of age) had EEs. WS was the commonest electroclinical syndrome followed by LGS in this study. These findings are similar to other studies [22] [31] [32]. Our study also observed that majority of children with WS had a history of perinatal asphyxia.

Classification under specific epileptic syndromes was difficult in $9.26 \%$ of cases and remained unclassified. Among them, the majority have a history of perinatal asphyxia, CNS infection, or septicemia. Most of them had predominantly generalized tonic-clonic and myoclonic seizures along with other seizure types and faced developmental regression after the onset of the seizure. EEG did not reveal any specific pattern. The majority showed multifocal, focal, and generalized epileptiform discharges.

The progression of EEs in children is variable and the prognosis does not depend on a specific factor. However, the electro-clinical syndrome gives some clues about the cause and outcome of the disease. It is useful to set up a treatment strategy as most of EEs are pharmacoresistant. Early, aggressive and multidisciplinary treatment approaches are needed for these children.

Therefore, the burden of EEs is huge in a country with limited resources like Bangladesh. It collectively exerts a profound financial toll on the families of affected children, healthcare providers, and the whole healthcare system. A high index of suspicion, proper EEG recordings, and appropriate analysis of EEG are mandatory for the early diagnosis of these patients. The knowledge of specific electro-clinical syndrome will help to treat these children early and appropriately which may improve the long-term outcome of these children with EEs.

This study was conducted in a single-center tertiary hospital and did not represent the actual situation of the country.

\section{Conclusion}

The frequency of EEs in infants and children is quite high. Perinatal asphyxia is an important cause. Most starts at an early age with a deleterious effect on the developing brain. Infantile spasms, myoclonic seizures are among the commonest seizure types. EEG in most cases shows multifocal epileptiform discharges in addition to other type-specific findings. WS and LGS constitute the major bulk of the EEs in children.

\section{Acknowledgements}

We would like to convey my sincere gratitude to the parents who agreed to let 
their children participate in the study. We also thank my colleagues for their assistance during data collection. The study was not funded by any authority.

\section{Authors' Contributions}

Bithi Debnath designed the study, performed the statistical analysis, wrote the protocol, and wrote the first draft of the manuscript. Mohammad Enayet Hussain reported all the EEG and managed the literature searches. RajibNayan Chowdhury and Narayan Saha managed the analyses of the study. All authors read and approved the final manuscript.

\section{Consent}

Written informed consent was taken from the parents or guardians before inclusion in the study.

\section{Ethical Approval}

Ethical clearance from the ethical review committee (ERC) of the hospital was taken before starting the study.

\section{Conflicts of Interest}

There is no conflict of interest with any of the authors of this article.

\section{References}

[1] Berg, A.T., Berkovic, S.F., Brodie, M.J., Buchhalter, J., Cross, J.H., van Emde Boas, W., Engel, J., French, J., Glauser, T.A., Mathern, G.W. and Moshé, S.L. (2010) Revised Terminology and Concepts for Organization of Seizures and Epilepsies: Report of the ILAE Commission on Classification and Terminology, 2005-2009. Epilepsia, 51, 676-685. https://doi.org/10.1111/j.1528-1167.2010.02522.x

[2] Jain, P., Sharma, S. and Tripathi, M. (2013) Diagnosis and Management of Epileptic Encephalopathies in Children. Epilepsy Research and Treatment, 2013, Article ID: 501981. https://doi.org/10.1155/2013/501981

[3] Dulac, O. (2001) Epileptic Encephalopathy. Epilepsia, 42, 23-26. https://doi.org/10.1046/j.1528-1157.2001.042suppl.3023.x

[4] Nabbout, R. and Dulac, O. (2003) Epileptic Encephalopathies: A Brief Overview. Journal of Clinical Neurophysiology, 20, 393-397. https://doi.org/10.1097/00004691-200311000-00002

[5] Oka, E., Ishida, S., Ohtsuka, Y. and Ohtahara, S. (1995) Neuroepidemiological Study of Childhood Epilepsy by Application of International Classification of Epilepsies and Epileptic Syndromes (ILAE, 1989). Epilepsia, 36, 658-661. https://doi.org/10.1111/j.1528-1157.1995.tb01042.x

[6] MacAllister, W.S. and Schaffer, S.G. (2007) Neuropsychological Deficits in Childhood Epilepsy Syndromes. Neuropsychology Review, 17, 427-444. https://doi.org/10.1007/s11065-007-9048-4

[7] Holmes, G.L. and Lenck-Santini, P.P. (2006) Role of Interictal Epileptiform Abnormalities in Cognitive Impairment. Epilepsy \& Behavior, 8, 504-515. https://doi.org/10.1016/j.yebeh.2005.11.014 
[8] Chugani, H.T. (1995) Infantile Spasms. Current Opinion in Neurology, 8, 139-144. https://doi.org/10.1097/00019052-199504000-00010

[9] Khan, N.Z., Muslima, H., Begum, D., Shilpi, A.S. and Akhter, S. (2010) Rapid Neurodevelopmental Assessment: Validation of a New Tool for Functional Evaluation of 0-24 Month Old Children in Bangladesh. Pediatrics, 125, 755-762. https://doi.org/10.1542/peds.2008-3471

[10] Khan, N.Z., Muslima, H., Shilpi, A.B., Begum, D., Parveen, M., Akter, N., Ferdous, S., Nahar, K., McConachie, H. and Darmstadt, G.L. (2013) Validation of Rapid Neurodevelopmental Assessment for 2- to 5-Year-Old Children in Bangladesh. Pediatrics, 131, 486-494. https://doi.org/10.1542/peds.2011-2421

[11] Kalra, V., Gulati, S., Pandey, R.M. and Menon, S. (2002) West Syndrome and Other Infantile Epileptic Encephalopathies-Indian Hospital Experience. Brain and Development, 24, 130-139. https://doi.org/10.1016/S0387-7604(02)00029-3

[12] Ohtahara, S., Ohtsuka, Y. and Oka, E. (1997) Epileptic Encephalopathies in Early Infancy. The Indian Journal of Pediatrics, 64, 603-612.

https://doi.org/10.1007/BF02726112

[13] Baram, T.Z. (1999) Myoclonus and Myoclonic Seizures. In: Swaiman, K.F. and Ashwal, S., Eds., Pediatric Neurology: Principles and Practice, 3rd Edition, Mosby Inc., St Louis, 668-675.

[14] Ohtsuka, Y., Ogino, T., Murakami, N., Mimaki, N., Kobayashi, K. and Ohtahara, S. (1986) Developmental Aspects of Epilepsy with Special Reference to Age-Dependent Epileptic Encephalopathy. Psychiatry and Clinical Neurosciences, 40, 307-313. https://doi.org/10.1111/j.1440-1819.1986.tb03152.x

[15] Udani, V.P., Dhamidharka, V., Nair, A. and Oka, M. (1993) Difficult to Control Epilepsy in Childhood-A Long Term Study of 123 Cases. Indian Pediatrics, 30, 1199-1206.

[16] Banu, S.H., Khan, N.Z., Hossain, M., Ferdousi, S., Boyd, S., Scott, R.C. and Neville, B. (2012) Prediction of Seizure Outcome in Childhood Epilepsies in Countries with Limited Resources: A Prospective Study. Developmental Medicine \& Child Neurology, 54, 918-924. https://doi.org/10.1111/j.1469-8749.2012.04325.x

[17] Seth, A., Aneja, S. and Taluja, V. (2001) Epileptic Encephalopathies of Early Childhood. Indian Pediatrics, 38, 390-396.

[18] Chandra, S. and Kumar, R. (2016) Clinico-Aetiological Profile and Outcome of West Syndrome from North India. Hong Kong Journal of Paediatrics (New Series), 21, 262-265.

[19] Kaushik, J.S., Patra, B., Sharma, S., Yadav, D. and Aneja, S. (2013) Clinical Spectrum and Treatment Outcome of West Syndrome in Children from Northern India. Seizure, 22, 617-621. https://doi.org/10.1016/j.seizure.2013.04.014

[20] Matsuo, A., Matsuzaka, T., Tsuru, A., Moriuchi, H., Nakashita, Y., Tanaka, S., Baba, C. and Tomimasu, K. (2001) Epidemiological and Clinical Studies of West Syndrome in Nagasaki Prefecture, Japan. Brain and Development, 23, 575-579. https://doi.org/10.1016/S0387-7604(01)00267-4

[21] Kalra, V.N. and Passi, G.R. (1998) Analysis of Childhood Epileptic Encephalopathies: Neuroradiologic Aspect of West Syndrome. Pediatric Neurology, 19, 1-8.

[22] Mackay, M.T., Weiss, S.K., Adams-Webber, T., Ashwal, S., Stephens, D., Ballaban-Gill, K., Baram, T.Z., Duchowny, M., Hirtz, D., Pellock, J.M. and Shields, W.D. (2004) Practice Parameter: Medical Treatment of Infantile Spasms: Report of the American Academy of Neurology and the Child Neurology Society. Neurology, 62, 1668-1681. https://doi.org/10.1212/01.WNL.0000127773.72699.C8 
[23] Tsuji, T., Okumura, A., Ozawa, H., Ito, M. and Watanabe, K. (2007) Current Treatment of West Syndrome in Japan. Journal of Child Neurology, 22, 560-564. https://doi.org/10.1177/0883073807302606

[24] Matsumoto, A., Watanabe, K., Negoro, T., Sugiura, M., Iwase, K., Hara, K. and Miyazaki, S. (1981) Infantile Spasms: Etiological Factors, Clinical Aspects, and Long Term Prognosis in 200 Cases. European Journal of Pediatrics, 135, 239-244. https://doi.org/10.1007/BF00442097

[25] Cusmai, R., Ricci, S., Pinard, J.M., Plouin, P., Fariello, G. and Dulac, O. (1993) West Syndrome Due to Perinatal Insults. Epilepsia, 34, 738-742. https://doi.org/10.1111/j.1528-1157.1993.tb00455.x

[26] Kalra, V. and Passi, G.R. (1998) Analysis of Childhood Epileptic Encephalopathies with Regard to Etiological and Prognostic Factors. Brain and Development, 20, 14-17. https://doi.org/10.1016/S0387-7604(97)00080-6

[27] Wallace, S.J. (1993) Seizures in Children. In: Laidlaw, J., Richens, A. and Chadwick, D., Eds., A Textbook of Epilepsy, 4th Edition, Churchill Livingstone, Edinburgh, 77-164.

[28] Kotagal, P. (1995) Multifocal Independent Spike Syndrome: Relationship to Hypsarrhythmia and the Slow Spike-Wave (Lennox-Gastaut) Syndrome. Clinical Electroencephalography, 26, 23-29. https://doi.org/10.1177/155005949502600105

[29] Yamatogi, Y. and Ohtahara, S. (2006) Multiple Independent Spike Foci and Epilepsy, with Special Reference to a New Epileptic Syndrome of "Severe Epilepsy with Multiple Independent Spike Foci”. Epilepsy Research, 70, S96-S104.

https://doi.org/10.1016/j.eplepsyres.2006.01.013

[30] Mohammad, Q.D., Saha, N.C., Alam, M.B., Hoque, S.A., Islam, A., Chowdhury, R.N., Hussain, M.E., Chowdhury, Y.S., Hossain, S., Chowdhury, M.A. and Rahman, M. (2020) Prevalence of Epilepsy in Bangladesh: Results from a National Household Survey. Epilepsia Open, 5, 526-536. https://doi.org/10.1002/epi4.12430

[31] Hwang, S.K. and Kwon, S. (2015) Early-Onset Epileptic Encephalopathies and the Diagnostic Approach to Underlying Causes. Korean Journal of Pediatrics, 58, 407. https://doi.org/10.3345/kjp.2015.58.11.407

[32] Alam, S. and Lux, A.L. (2012) Epilepsies in Infancy. Archives of Disease in Childhood, 97, 985-992. https://doi.org/10.1136/archdischild-2011-301119 